\title{
Quantum Phase Transition in the Four-Spin Exchange Antiferromagnet
}

\author{
Valeri N. Kotov, Dao-Xin Yao, A. H. Castro Neto, and D. K. Campbell \\ Department of Physics, Boston University, 590 Commonwealth Avenue, Boston, MA 02215
}

\begin{abstract}
We study the $S=1 / 2$ Heisenberg antiferromagnet on a square lattice with nearest-neighbor and plaquette fourspin exchanges (introduced by A.W. Sandvik, Phys. Rev. Lett. 98, 227202 (2007).) This model undergoes a quantum phase transition from a spontaneously dimerized phase to Néel order at a critical coupling. We show that as the critical point is approached from the dimerized side, the system exhibits strong fluctuations in the dimer background, reflected in the presence of a low-energy singlet mode, with a simultaneous rise in the triplet quasiparticle density. We find that both singlet and triplet modes of high density condense at the transition, signaling restoration of lattice symmetry. In our approach, which goes beyond mean-field theory in terms of the triplet excitations, the transition appears sharp; however since our method breaks down near the critical point, we argue that we cannot make a definite conclusion regarding the order of the transition.
\end{abstract}

PACS numbers: $75.10 . J \mathrm{~m}, 75.30 . \mathrm{Kz}, 75.50 . \mathrm{Ee}$

\section{INTRODUCTION}

Problems related to quantum criticality in quantum spin systems are of both fundamental and practical importance ${ }^{1}$. Numerous materials, such as Mott insulators, exhibit either antiferromagnetic (Néel) order or quantum disordered (spin gapped) ground state depending on the distribution of Heisenberg exchange couplings and geometry. External perturbations (such as doping or frustration) can also cause quantum transitions between these phases. Systems with spin 1/2 are indeed the most interesting as they are the most susceptible to such transitions. It is well understood that the quantum transition between a quantum disordered and a Néel phase is in the $O(3)$ universality class ${ }^{1}$, where a triplet state condenses at the quantum critical point (QCP).

A recent exciting development in our theoretical understanding of QCPs originated from the proposal that if the quantum disordered (QD) phase spontaneously breaks lattice symmetries (e.g. is characterized by spontaneous dimer order), and the transition is of second order, then exactly at the QCP spinon deconfinement occurs, i.e. the excitations are fractionalized ${ }^{2}$. It is assumed that the Hamiltonian itself does not break the lattice symmetries (i.e. does not have "trivial" dimer order caused by some exchanges being stronger than the others). We use the terms "dimer order" and "valence bond solid (VBS) order" interchangeably. It is expected that the dimer order vanishes exactly at the point where Néel order appears, i.e. there is no coexistence between the two phases. Deconfinement thus is intimately related to disappearance of VBS order; indeed if the latter persisted in the Néel phase it would be impossible to isolate a spinon, as "pairing" would always take place. Spontaneous VBS order driven by frustration has been a common theme in quantum antiferromagnetism ${ }^{3}$, although its presence and the nature of criticality in specific models, such as the 2D squarelattice frustrated Heisenberg antiferromagnet, is still somewhat controversial ${ }^{4}$. It would be particularly useful to apply unbiased numerical approaches, such as the Quantum Monte Carlo (QMC) method, to study frustrated spin models; however due to the fatal "sign" problem ${ }^{5}$, frustrated Heisenberg systems are beyond the QMC reach.
In a recent study, the QMC method was applied to a fourspin exchange quantum spin model without frustration, which was shown to exhibit columnar dimer VBS order and a magnetically ordered phase with a deconfined QCP separating them ${ }^{6}$. These conclusions were later confirmed by further QMC studies? 7 . Extensions of the model, which include for example additional (six-spin) interactions, provide additional support for a continuous QCP ${ }^{8}$. A different VBS pattern (plaquette order) was also proposed for the four-spin exchange $\operatorname{model}^{9}$. At the same time, the nature of the quantum phase transition was challenged in Refs. [10.11], where arguments were given that the transition is in fact of (weakly) first order.

It is the objective of the present work to study the Sandvik model ${ }^{6}$, by approaching the quantum transition from the dimer VBS phase. Our approach uses as a starting point a symmetry broken state (i.e. one out of four degenerate VBS configurations), and we thus must search for signatures that the system attempts to restore the lattice symmetry at the QCP. Even though full restoration is impossible within the present framework, we find a QCP characterized by condensation of triplet modes of high density; this is in contrast to the conventional situation when the condensing particles are in the dilute Bose gas limit. The high density itself is due to the presence of a singlet mode that condenses at the QCP, and reflects the strong fluctuations of the background dimer order. The above effects lead to the vanishing of the VBS order parameter; at the same time our method, which accounts for the strong fluctuations, leads to a rather sharp phase transition. It appears that we cannot draw a definite conclusion about the order of the transition because in the vicinity of the QCP the triplon density increases uncontrollably, suggesting that other states (such a plaquette states and larger clusters) are strongly admixed into the ground state. This is generally expected in a situation where the lattice symmetry is restored at the quantum critical point.

The model under consideration is

$$
H=J \sum_{\langle a, b\rangle} \mathbf{S}_{a} \cdot \mathbf{S}_{b}-K \sum_{a, b, c, d}\left(\mathbf{S}_{a} \cdot \mathbf{S}_{b}\right)\left(\mathbf{S}_{c} \cdot \mathbf{S}_{d}\right),
$$

where $J>0, K>0$, and all spins are $S=1 / 2$. Consider the numbers 1,2,3,4 in Fig. 1 The summation in the fourspin term is over indexes $(a, b)=(1,2),(c, d)=(3,4)$ and 


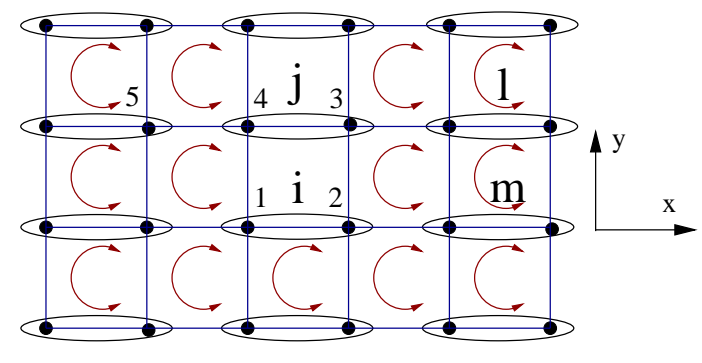

FIG. 1: (Color online) Dimer pattern in the quantum disordered (VBS) phase, $K / J>(K / J)_{c}$.

$(a, b)=(1,4),(c, d)=(2,3)$ on a given plaquette, and then summation is made over all plaquettes 12 . The range of parameters explored in Ref.[6] is $K / J \leq 2$, and the QCP is at $(K / J)_{c} \approx 1.85$. Our coupling notation is slightly different from the one used in Refs. [6.7]; the coupling $K$ is related to the parameter $Q^{6,7}$ via $K=Q /(1+Q /(2 J))$, and the critical point in that notation is $(Q / J)_{c} \approx 25$. The dimerization pattern is proposed to be of the "columnar" type, as shown in Fig. 11 Four such configurations exist. We will assume a configuration of this type, will show that it is stable at $K / J \gg 1$, and will then search for an instability towards the Néel state as $K / J$ decreases.

The rest of the paper is organized as followed. In Section II we present results based on the mean-field approach in terms of the dimer (triplon) operators. In Section III we extend our treatment beyond mean-field, and even further in Section IV, where we also take into account low-energy singlet twotriplon excitations. Section V contains our conclusions.

\section{MEAN-FIELD TREATMENT}

We start by rewriting Eq. (1) in the the bond-operator representation ${ }^{13}$, where on a dimer $\mathbf{i}$, the two spins forming it are expressed as: $S_{1,2}^{\alpha}=\frac{1}{2}\left( \pm s_{\mathbf{i}}^{\dagger} t_{\mathbf{i} \alpha} \pm t_{\mathbf{i} \alpha}^{\dagger} s_{\mathbf{i}}-i \epsilon_{\alpha \beta \gamma} t_{\mathbf{i} \beta}^{\dagger} t_{\mathbf{i} \gamma}\right)$, and $s_{\mathbf{i}}^{\dagger}, t_{\mathbf{i} \alpha}^{\dagger}, \alpha=x, y, z$ create a singlet and triplet of states. We refer to the triplet $(\mathrm{S}=1)$ quasiparticle, $t_{\mathbf{i} \alpha}^{\dagger}$, as "triplon". The bold indexes $\mathbf{i}, \mathbf{j}, \mathbf{m}, \mathbf{l}$ label the dimers (see Fig. 1). Summation over repeated Greek indexes is assumed, unless indicated otherwise.

The hard-core constraint, $s_{\mathbf{i}}^{\dagger} s_{\mathbf{i}}+t_{\mathbf{i} \alpha}^{\dagger} t_{\mathbf{i} \alpha}=1$, must be enforced on every site, which at the mean-field (MF) level can be done by introducing a term in the Hamiltonian, $-\mu \sum_{\mathbf{i}}\left(s^{2}+\right.$ $\left.t_{\mathbf{i} \alpha}^{\dagger} t_{\mathbf{i} \alpha}-1\right)$. Then $\mu$ and the (condensed) singlet amplitude $s \equiv\left\langle s_{\mathbf{i}}\right\rangle$, are determined by the MF equations ${ }^{13}$. We obtain at the quadratic level, in momentum representation:

$$
H_{2}=\sum_{\mathbf{k}, \alpha}\left\{A_{\mathbf{k}} t_{\mathbf{k} \alpha}^{\dagger} t_{\mathbf{k} \alpha}+\frac{B_{\mathbf{k}}}{2}\left(t_{\mathbf{k} \alpha}^{\dagger} t_{-\mathbf{k} \alpha}^{\dagger}+\text { h.c. }\right)\right\}
$$

where

$$
\begin{aligned}
& A_{\mathbf{k}}=J / 4-\mu+s^{2}\left(\xi_{\mathbf{k}}^{-}+K / 2\right)+s^{4} \Sigma(\mathbf{k}), \\
& B_{\mathbf{k}}=s^{2} \xi_{\mathbf{k}}^{+}+s^{4} \Sigma(\mathbf{k}), \\
& \xi_{\mathbf{k}}^{ \pm}=-(J / 2) \cos k_{x}+(J \pm K / 4) \cos k_{y} .
\end{aligned}
$$

The four-spin interaction from (1) acting between two dimers (e.g. $\mathbf{i}, \mathbf{j}$ in Fig. 1) contributes to the "on-site" gap and hopping $\left(\xi_{\mathbf{k}}^{-}\right)$via $A_{\mathbf{k}}$, as well as to the quantum fluctuations term $B_{\mathbf{k}}$. The part involving four dimers has been split in a mean-field fashion, leading to the Hartree-Fock self-energy

$$
-\Sigma(\mathbf{k}) / K=2 \Sigma_{x} \cos k_{x}+2 \Sigma_{y} \cos k_{y}+\Sigma_{x y} \cos k_{x} \cos k_{y},
$$

with

$$
\Sigma_{x}=\frac{1}{3} \sum_{\alpha}\left\langle t_{\mathbf{i} \alpha}^{\dagger} t_{\mathbf{m} \alpha}+t_{\mathbf{i} \alpha}^{\dagger} t_{\mathbf{m} \alpha}^{\dagger}\right\rangle
$$

where $\mathbf{i}, \mathbf{m}$ are neighboring dimers in the $x$ (horizontal) direction (Fig. 1), and similarly for the $y$ and the diagonal contributions. The triplon dispersion is $\omega(\mathbf{k})=\sqrt{A_{\mathbf{k}}^{2}-B_{\mathbf{k}}^{2}}$, and has a minimum at the Néel ordering wave-vector $\mathbf{k}_{A F}=(0, \pi)$ (since we work on a dimerized lattice). The ground state energy is then easily computed,

$$
E_{G S}=E_{0}+\left\langle H_{2}\right\rangle
$$

where

$$
\begin{aligned}
E_{0} / N= & -\frac{3}{4}\left(J s^{2}+K s^{4}\right)+\mu\left(-s^{2}+1\right)+ \\
& 3 K s^{4}\left(\Sigma_{x}^{2}+\Sigma_{y}^{2}+\frac{1}{2} \Sigma_{x y}^{2}\right)
\end{aligned}
$$

and

$$
\left\langle H_{2}\right\rangle=\frac{3}{2} \sum_{\mathbf{k}}\left(\omega(\mathbf{k})-A_{\mathbf{k}}\right) .
$$

The mean-field equations require a numerical minimization of $E_{G S}$ with respect to the parameters $\left\{\mu, s, \Sigma_{x}, \Sigma_{y}, \Sigma_{x y}\right\}$. This amounts to the self-consistent Hartree-Fock approximation for $\Sigma(\mathbf{k})$. The result for the triplon gap $\Delta=\omega\left(\mathbf{k}_{A F}\right)$ is presented in Fig. 2 (black curve).

The MF result $(K / J)_{c} \approx 0.6$ substantially underestimates the location of the critical point, compared to the the QMC calculations, where $(K / J)_{c} \approx 1.85^{6,7}$. Interestingly, if one solves the MF equations ignoring both the hard core and the $\Sigma(\mathbf{k})$, one finds $(K / J)_{c}=1$. Physically, in the full MF, the hard core contribution increases the gap (and hence the stability of the dimer phase) while at the same time suppressing the antiferromagnetic fluctuations (which favor the Néel state).

We also note that a recent (hierarchical) MF treatment based on the plaquette ground state also underestimates very strongly the QCP location $\left((K / J)_{c} \approx 1^{9}\right)$, similarly to our result. In our view this means that both mean field approaches are not sufficient to attack the present problem, where fluctuations are apparently very strong. We choose to accept that the numerical QMC result gives the most accurate determination of the QCP, and therefore in what follows we extend our treatment in several directions beyond mean-field theory. 


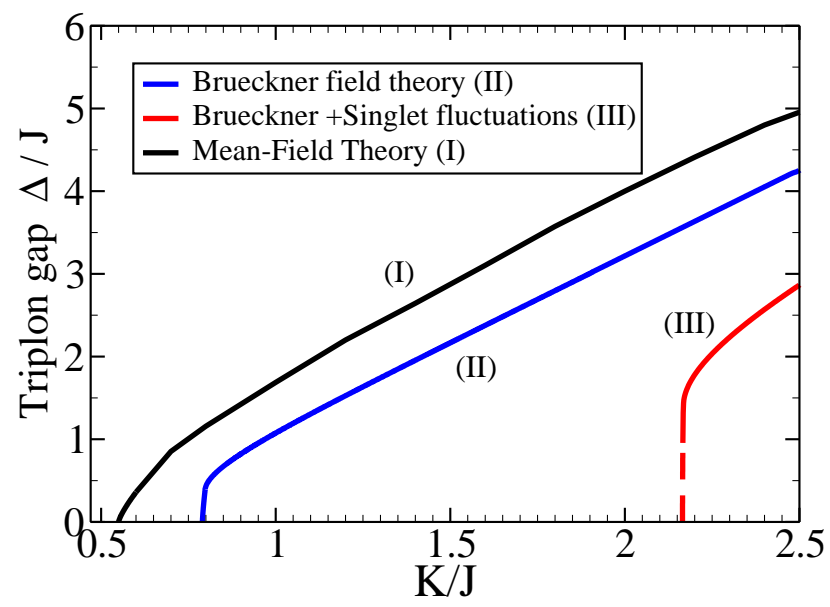

FIG. 2: (Color online) Triplon excitation gap $\Delta=\omega\left(\mathbf{k}_{A F}\right)$ in various approximations. The point $\Delta \rightarrow 0$ corresponds to transition to the Néel phase.

\section{BEYOND MEAN-FIELD: THE DILUTE TRIPLON GAS APPROXIMATION}

A more accurate treatment of fluctuations is possible by taking into account the hard-core constraint beyond meanfield. One can set the singlet amplitude $s=1$ in the previous formulas, but introduce an infinite on-site repulsion between the triplons, $U \sum_{\mathbf{i}, \alpha \beta} t_{\alpha \mathbf{i}}^{\dagger} t_{\beta \mathbf{i}}^{\dagger} t_{\beta \mathbf{i}} t_{\alpha \mathbf{i}}, U \rightarrow \infty$. As long as the triplon density (determined by the quantum fluctuations) is low, an infinite repulsion corresponds to a finite scattering amplitude between excitations and can be calculated by resumming ladder diagrams for the scattering vertex ${ }^{14}$. This leads to the effective triplon-triplon vertex $\Gamma(\mathbf{k}, \omega)$ which was previously calculated ${ }^{15}$ :

$$
\Gamma^{-1}(\mathbf{k}, \omega)=\sum_{\mathbf{q}} \frac{u_{\mathbf{q}}^{2} u_{\mathbf{k}-\mathbf{q}}^{2}}{\omega(\mathbf{q})+\omega(\mathbf{k}-\mathbf{q})-\omega}+\left\{\begin{array}{c}
u \rightarrow v \\
\omega \rightarrow-\omega
\end{array}\right\}
$$

This vertex in turn affects the triplon dispersion via (what we call) the Brueckner self-energy 15 :

$$
\Sigma_{B}(\mathbf{k}, \omega)=4 \sum_{\mathbf{q}} v_{\mathbf{q}}^{2} \Gamma(\mathbf{k}+\mathbf{q}, \omega-\omega(\mathbf{q})) .
$$

The corresponding parameters in the quadratic Hamiltonian (2) in this case are

$$
\begin{aligned}
& A_{\mathbf{k}}=J+2 K\left(1-4 n_{t} / 3\right)+\xi_{\mathbf{k}}^{-}+\Sigma(\mathbf{k})+\Sigma_{B}(\mathbf{k}, 0) \\
& B_{\mathbf{k}}=\xi_{\mathbf{k}}^{+}+\Sigma(\mathbf{k}) .
\end{aligned}
$$

The Bogolubov coefficients are defined in the usual way $u_{\mathbf{k}}^{2}=1 / 2+A_{\mathbf{k}} /(2 \omega(\mathbf{k}))=1+v_{\mathbf{k}}^{2}$. The various terms in $\Sigma(\mathbf{k})$ can be expressed through them: for example $\Sigma_{x}=$ $\sum_{\mathbf{k}}\left(v_{\mathbf{k}}^{2}+v_{\mathbf{k}} u_{\mathbf{k}}\right) \cos k_{x}$, and so on. The density of triplons is $n_{t}=\left\langle t_{\mathbf{i} \alpha}^{\dagger} t_{\mathbf{i} \alpha}\right\rangle=3 \sum_{\mathbf{k}} v_{\mathbf{k}}^{2}$. In addition, the renormalization of the quasiparticle residue, $Z_{\mathbf{k}}^{-1}=1-\partial \Sigma_{B}(\mathbf{k}, 0) / \partial \omega$,

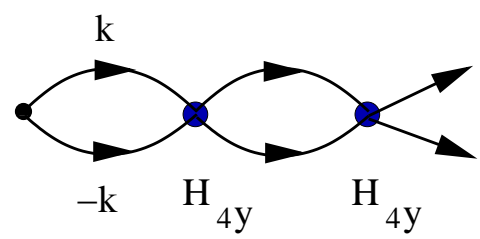

FIG. 3: Renormalization of quantum fluctuations by resummation of a ladder series, with (13) at the vertices.

implies the replacement $u_{\mathbf{k}} \rightarrow \sqrt{Z_{\mathbf{k}}} u_{\mathbf{k}}, v_{\mathbf{k}} \rightarrow \sqrt{Z_{\mathbf{k}}} v_{\mathbf{k}}$ in all the formulas ${ }^{15}$, and the renormalized spectrum $\omega(\mathbf{k})=$ $Z_{\mathbf{k}} \sqrt{A_{\mathbf{k}}^{2}-B_{\mathbf{k}}^{2}}$.

An iterative numerical evaluation of the spectrum using the above equations, which amounts to solution of the Dyson equation, leads to the result shown in Fig. 2(blue curve). The above approach appears to be well justified since the quasiparticle density $n_{t}<0.1$. The resulting critical point is still in the "weak-coupling" regime $K / J<1$, with about $100 \%$ deviation from the $\mathrm{QMC}$ result $\left((K / J)_{c} \approx 1.85\right)$. This suggests that the on-site triplon fluctuations are not the dominant cause for the disagreement with the QMC results; thus we proceed to include two-particle fluctuations (in the triplon language), which amounts to including dimer-dimer correlations.

\section{STRONG FLUCTUATIONS IN THE SINGLET BACKGROUND: QCP BEYOND THE DILUTE TRIPLON GAS APPROXIMATION}

It is clear that "non-perturbative" effects are responsible for driving the QCP towards the "strong-coupling" region $K / J \sim$ 2. To proceed we make two improvements to the previous low-density, weak-coupling theory.

First, we take into account fluctuations in the singlet background, i.e. the manifold on which the triplons are built and interact. The main effect originates from the action of the four-spin $K$-term from (1) on two dimers, e.g. i, $\mathbf{j}$ in Fig. 1 . Part of this action has led to the on-site gap $2 K$ in (12), favoring dimerization. However, a strong attraction between the two dimers is also present, since the $K$-term is symmetric with respect to the index pair exchange $(1,2)(3,4) \leftrightarrow(1,4)(2,3)$, leading to a "plaquettization" tendency as well. In the triplon language this is manifested by formation of bound states of two triplons, due to their nearest-neighbor interactions

$$
\begin{aligned}
H_{4, y}= & \sum_{\langle\mathbf{i}, \mathbf{j}\rangle_{y}, \alpha \beta}\left\{\gamma_{1} t_{\alpha \mathbf{i}}^{\dagger} t_{\beta \mathbf{j}}^{\dagger} t_{\beta \mathbf{i}} t_{\alpha \mathbf{j}}+\gamma_{2} t_{\alpha \mathbf{i}}^{\dagger} t_{\alpha \mathbf{j}}^{\dagger} t_{\beta \mathbf{i}} t_{\beta \mathbf{j}}\right. \\
& \left.+\gamma_{3} t_{\alpha \mathbf{i}}^{\dagger} t_{\beta \mathbf{j}}^{\dagger} t_{\alpha \mathbf{i}} t_{\beta \mathbf{j}}\right\}, \\
\gamma_{1}= & -\frac{K}{8}+\frac{J}{2}, \gamma_{2}=-\frac{K}{8}-\frac{J}{2}, \gamma_{3}=-\frac{5 K}{4} .
\end{aligned}
$$

We also checked that on the perturbative (Hartree-Fock) level, the effect of this term on equations (3) and (12) was negligible (and we did not write it explicitly).

An intuitive way of taking into account the effect of twotriplon bound states (with total spin $S=0$ ) on the one-triplon 

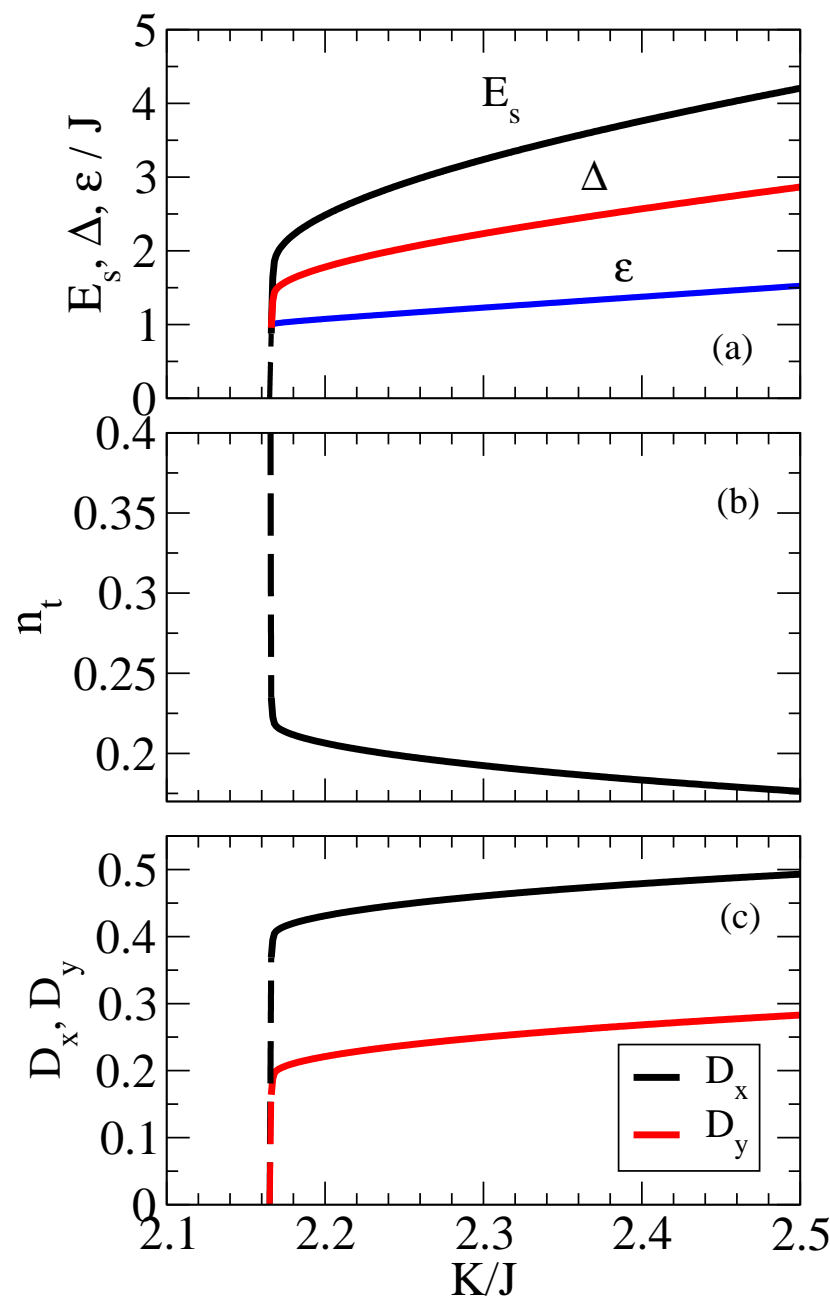

FIG. 4: (Color online) (a.) Singlet bound state energy $E_{s}$ (black), binding energy $\epsilon=2 \Delta-E_{s}$ (blue), and the triplon gap $\Delta$ (red). (b.) Triplon density $n_{t}$. (c.) Dimer order parameters. Dashed parts of the lines represent points corresponding to rapid growth of the quasiparticle density.

spectrum, is to work in the "local" approximation. This means effectively neglecting the triplon dispersion and directly evaluating the ladder series that renormalizes the quantum fluctuation term $B_{\mathbf{k}}$ in (2), corresponding to emission of a pair of triplons with zero total momentum. This is illustrated graphically in Fig. 3, with the result

$$
\begin{gathered}
B_{\mathbf{k}}=-\frac{J}{2} \cos k_{x}+\left(\frac{J+K / 4}{1-\frac{|\gamma|}{|\Delta E|}}\right) \cos k_{y}+\Sigma(\mathbf{k}) \\
\gamma \equiv \gamma_{1}+3 \gamma_{2}+\gamma_{3}=-J-\frac{7}{4} K
\end{gathered}
$$

where $\gamma$ is the effective attraction of two triplons with total $S=0$, and

$$
\Delta E=2 J+\frac{11}{4} K
$$

is the energy of two (non-interacting) triplons on adjacent sites. This calculation is justified for $K / J \gg 1$ and leads to an increase of the quantum fluctuations, and from there to almost doubling of the triplon density $n_{t}$ (see Fig. 4 below). It contributes significantly to the shift of the QCP.

We can go beyond the "local" approximation by solving the Bethe-Salpeter equation for the bound state, formed due to the attraction (13), and taking into account the full triplon dispersion. The equation for the singlet bound state energy $E_{s}(\mathbf{Q})$, corresponding to total pair momentum $\mathbf{Q}$ is

$$
1=2 \gamma \sum_{\mathbf{q}} \frac{u_{\mathbf{q}}^{4} \cos ^{2} q_{y}}{E_{s}(\mathbf{Q})-\omega(\mathbf{Q} / 2+\mathbf{q})-\omega(\mathbf{Q} / 2-\mathbf{q})}
$$

Here we have, for simplicity, written only the main contribution to pairing (Eq. (13)) in the limit $K / J \gg 1$, and have neglected the on-site repulsion (which leads to slightly diminished pairing), as well as small pairing due to the exchange $J$ from dimers in the $x$-direction on Fig. 11, It is easily seen that the lowest energy corresponds to $\mathbf{Q}=0$; we define from now on $E_{s} \equiv E_{s}(\mathbf{Q}=0)$. The binding energy is $\epsilon=2 \Delta-E_{s}$, where $\Delta$ is the one-particle gap. The bound state wave-function corresponding to $E_{s}$ is $|\Psi\rangle=\sum_{\alpha, \mathbf{i}, \mathbf{j}, q_{y}} \Psi_{q_{y}} e^{i q_{y}(\mathbf{i}-\mathbf{j})} t_{\alpha \mathbf{i}}^{\dagger} t_{\alpha \mathbf{j}}^{\dagger}|0\rangle$. In the "local" limit (nearest-neighbor pairing), $\Psi_{q_{y}}=\sqrt{2} \cos q_{y}$.

Second, we have made subtle changes to the resummation procedure concerning the quasiparticle renormalization $Z$, based on both formal and physical grounds. On the one hand it is clear that in the Brueckner approximation (Eq. (11)), where the self energy is linear in the density $\left(\Sigma_{B} \propto n_{t}\right)$, the dependence of the vertex $\Gamma^{-1}$ on density is beyond the accuracy of the calculation, meaning one can put $u_{\mathbf{q}}=1, v_{\mathbf{q}}=0$ in (10), instead of determining them self-consistently. This leads to a decreased influence of the hard-core $\Sigma_{B}$ (which favors the dimer state) on the Hartree-Fock self-energy $\Sigma(\mathbf{k})$ from (4) (which favors the Néel state). It is indeed the mutual interplay between $\Sigma_{B}>0$ and $\Sigma(\mathbf{k})<0$, that determines the exact location of the QCP in the course of the Dyson's equation iterative solution. While in the "weak-coupling" regime $K / J<1, \Sigma_{B}$ always dominates, in the "strong-coupling" region $K / J>2, \Sigma(\mathbf{k})$ starts playing a significant role, since parametrically $\Sigma \propto K n_{t}$. It is physically consistent that in the region where singlet fluctuations in the dimer background are strong, the hard-core effect is less important, i.e. in effect the kinematic hard-core constraint is "relaxed". We also observe that in typical models with QCP driven by explicit dimerization, such as the bilayer model, the described difference in approximation schemes makes a very small difference on the location of the $\mathrm{QCP}^{16}$, since those models are always in the "weak-coupling" regime, dominated by the hard-core repulsion of excitations on a non-fluctuating dimer configuration. The purpose of the above rather technical diversion is to emphasize that care has been taken to take into account as accurately as possible the effect of the (low-energy) two-particle spectrum on the one-particle triplon gap.

Our results are summarized in Fig. 4 and Fig. 2(red line) for the gap. The critical point is shifted towards $(K / J)_{c} \approx 2.16$ (in much better agreement with QMC data), with a very 
strong increase of the density towards $K_{c}$. This translates into a decrease of the dimer order, as measured by the two dimer order parameters that we compute from the expressions: $D_{x}=\left|\left\langle\mathbf{S}_{3} \cdot \mathbf{S}_{4}\right\rangle-\left\langle\mathbf{S}_{5} \cdot \mathbf{S}_{4}\right\rangle\right|=\left|-\frac{3}{4}+n_{t}+\frac{3}{4} \Sigma_{x}\right|$, $D_{y}=\left|\left\langle\mathbf{S}_{3} \cdot \mathbf{S}_{4}\right\rangle-\left\langle\mathbf{S}_{1} \cdot \mathbf{S}_{4}\right\rangle\right|=\left|-\frac{3}{4}+n_{t}-\frac{3}{4} \Sigma_{y}\right|$. The spins are labeled as in Fig. 1. The singlet bound state energy $E_{s}(0)$ also tends towards zero at the QCP, with the corresponding binding energy remaining quite large $\epsilon / J \approx 1$. All these effects point towards a tendency of the system to restore the lattice symmetry, although it is certainly clear that as the critical point is approached, our approximation scheme (low density of quasiparticles) breaks down (dashed lines on figures). We should point out that the sharpness of variation near $K_{c}$ is not due to divergence in any of the self-energies but is a result of rapid cancellation at high orders (i.e. iterations in the Dyson equation). In fact cutting off our iterative procedure at finite order gives a smooth curve, suggesting that additional classes of diagrams become important (although in practice their classification is an insurmountable task). The merger of singlet and triplet modes, which we find near the QCP, in principle reflects a tendency towards quasiparticle fractionalization (spinon deconfinement) and is also found in the 1D Heisenberg chain with frustration ${ }^{17}$, where spinons are always deconfined.

Since we are now dealing with a situation where the density is not very small $n_{t} \approx 0.2$, it is prudent to check how the next order in the density may affect the above results. For example at second order in the density, the self-energy $\Sigma(\mathbf{k})$ changes by amount $\delta \Sigma(\mathbf{k})$, i.e. one has to add this contribution to the right hand side of Eq.(12), namely:

$$
A_{\mathbf{k}} \rightarrow A_{\mathbf{k}}+\delta \Sigma(\mathbf{k}), \quad B_{\mathbf{k}} \rightarrow B_{\mathbf{k}}+\delta \Sigma(\mathbf{k}) .
$$

We have found

$$
\begin{aligned}
\delta \Sigma(\mathbf{k})= & -2 K\left(Q_{x}^{2}-P_{x}^{2}\right) \cos k_{x}+ \\
& +2 K\left(Q_{y}^{2}-P_{y}^{2}\right) \cos k_{y}- \\
& -K\left(Q_{x y}^{2}-P_{x y}^{2}\right) \cos k_{x} \cos k_{y},
\end{aligned}
$$

and the following definitions are used:

$$
\begin{aligned}
P_{x} & =(1 / 3) \sum_{\alpha}\left\langle t_{\mathbf{i} \alpha}^{\dagger} t_{\mathbf{m} \alpha}\right\rangle=\sum_{\mathbf{k}} v_{\mathbf{k}}^{2} \cos k_{x} \\
Q_{x} & =(1 / 3) \sum_{\alpha}\left\langle t_{\mathbf{i} \alpha} t_{\mathbf{m} \alpha}\right\rangle=\sum_{\mathbf{k}} u_{\mathbf{k}} v_{\mathbf{k}} \cos k_{x},
\end{aligned}
$$

and similarly for the other directions, for example $P_{y}=$ $\sum_{\mathbf{k}} v_{\mathbf{k}}^{2} \cos k_{y}, P_{x y}=\sum_{\mathbf{k}} v_{\mathbf{k}}^{2} \cos k_{x} \cos k_{y}$, etc. After including these expressions in our numerical iterative procedure, we have found that the QCP is shifted by a very small amount, and the overall picture, as summarized in Fig. 4 and Fig. 2 (red line), still stands.

\section{v. CONCLUSIONS}

In conclusion, we have shown that the QCP between the Néel and the dimer state in the model (1) is of unconven- tional nature, in the sense that it is characterized by the presence of both triplet and singlet low-energy modes. Near the QCP, whose location $\left((K / J)_{c} \approx 2.16\right)$ we find in fairly good agreement with recent QMC studies, the system exhibits: (1.) Strong rise of the triplon excitation density, due to increased quantum fluctuations, (2.) Corresponding strong decrease (and ultimately vanishing) of the dimer order at the QCP (3.) Vanishing of a singlet energy scale, related to the destruction of the dimer "columns" in Fig. 11 The above effects are all related and influence strongly one another, ultimately meaning that the QCP reflects strong fluctuations and can not be described in a mean-field theory framework. These results also suggest a desire of the system to restore the lattice symmetry at the QCP, as found in the QMC studies ${ }^{6}$.

At the same time all our improvements beyond mean-field theory have also resulted in a very sharp transition, which appears to be first order. However in our view our approach is not capable of addressing correctly the issue of the order of the phase transition, basically because once we take the strong (inter) dimer fluctuations in to account, the triplon density starts rising quickly beyond control. This is in a certain sense natural in a situation where the system wants to restore the lattice symmetry at the QCP and thus the ground state acquires strong admixture of plaquette, etc. fluctuations as the dimers begin to "disappear." This is also manifested in the fact that our procedure is sensitive to the number of iterations in the Dyson equation; all presented results are for an "infinite" number of iterations, so that a fixed point is reached, but cutting off the procedure results in a smoother behavior and a shift of the QCP, which becomes iteration dependent. We have not previously encountered such volatile behavior in any other spin model with a dimer to magnetic order transition. Since iterations translate into accounting of more and more fluctuations, the sensitivity of the results seems to mean that the situation starts spiraling out of control near the QCP, quite likely because classes of fluctuations become important that are not included in the dimer description, such as longer range correlations, etc. All this suggests that the triplon quasiparticle description breaks down near the QCP which indeed appears natural in a model where spinon deconfinement is expected to take place at the $\mathrm{QCP}^{6}$. On the other hand, if we put aside the arguments that our approach is not reliable near the QCP, the natural conclusion would be that the transition is first order.

\section{Acknowledgments}

We are grateful to A. W. Sandvik, K. S. D. Beach, S. Sachdev, and O. P. Sushkov for numerous stimulating discussions. A.H.C.N. was supported through NSF grant DMR0343790; V.N.K., D.X.Y., and D.K.C. were supported by Boston University. 
1 S. Sachdev, Quantum Phase Transitions (Cambridge University Press, Cambridge, 1999).

2 T. Senthil, A. Vishwanath, L. Balents, S. Sachdev, and M. P. A. Fisher, Science 303, 1490 (2004); T. Senthil, L. Balents, S. Sachdev, A. Vishwanath, and M. P.A. Fisher, Phys. Rev. B 70, 144407 (2004).

3 S. Sachdev and N. Read, Int. J. Mod. Phys. B 5, 219 (1991).

${ }^{4}$ R. R. P. Singh, Z. Weihong, C. J. Hamer, and J. Oitmaa, Phys. Rev. B 60, 7278 (1999); L. Capriotti, F. Becca, A. Parola, and S. Sorella, Phys. Rev. Lett. 87, 097201 (2001); M. Mambrini, A. Läuchli, D. Poilblanc, and F. Mila, Phys. Rev. B 74, 144422 (2006).

5 P. Henelius and A. W. Sandvik, Phys. Rev. B 62, 1102 (2000).

${ }^{6}$ A. W. Sandvik, Phys. Rev. Lett. 98, 227202 (2007).

${ }^{7}$ R. G. Melko and R. K. Kaul, Phys. Rev. Lett. 100, 017203 (2008).

8 J. Lou, A. W. Sandvik, and N. Kawashima, arXiv:0908.0740

${ }^{9}$ L. Isaev, G. Ortiz, and J. Dukelsky, arXiv:0903.1630.

${ }^{10}$ A. B. Kuklov, M. Matsumoto, N. V. Prokof'ev, B. V. Svistunov, and M. Troyer, Phys. Rev. Lett. 101, 050405 (2008).

11 F.-J. Jiang, M. Nyfeler, S. Chandrasekharan, and U.-J. Wiese, J. Stat. Mech., P02009 (2008).

12 The possibility of four-spin exchange induced dimerization has been discussed in the context of the full ring exchange, of which the interaction (1) is part; see e.g. A. Läuchli, J. C. Domenge, C. Lhuillier, P. Sindzingre, and M. Troyer, Phys. Rev. Lett. 95, 137206 (2005).

13 S. Sachdev and R. N. Bhatt, Phys. Rev. B 41, 9323 (1990).

14 A. L. Fetter and J. D. Walecka, Quantum Theory of Many-Particle Systems (Dover Publications, Mineola, NY, 2003).

15 V. N. Kotov, O. P. Sushkov, Z. Weihong, and J. Oitmaa, Phys. Rev. Lett. 80, 5790 (1998).

${ }^{16}$ P. V. Shevchenko, A. W. Sandvik, and O. P. Sushkov, Phys. Rev. B 61, 3475 (2000).

17 W. H. Zheng, C. J. Hamer, R. R. P. Singh, S. Trebst, and H. Monien, Phys. Rev. B 63, 144411 (2001); ibid. 63, 144410 (2001). 